\title{
The Timed Up and Go Test is Related to Quantitative Ultrasound Parameters of Bone Strength in Japanese Community-Dwelling Elderly Women
}

\author{
NAOTO Kamide, RPT, MS ${ }^{1,2)}$, Yoshitaka ShIBA, RPT, $\mathrm{PhD}^{1,3)}$, KATsura KoIDE, RPT ${ }^{4)}$, \\ Hiroshi Haga, PhD ${ }^{3)}$, Hiroshi Shibata, MD, $\mathrm{PhD}^{3)}$ \\ ${ }^{1)}$ School of Allied Health Sciences, Kitasato University: 1-15-1 Kitasato, Sagamihara, \\ Kanagawa 228-8555, Japan. TEL: +81 42-778-9693 \\ ${ }^{2)}$ Major in Gerontology, Graduate School of J.F. Oberlin University \\ 3) J.F.Oberlin University Institution of Aging and Human Development \\ ${ }^{4}$ Graduate School of Medical Science, Kitasato University
}

\begin{abstract}
Purpose] The timed up and go test (TUGT) requires no special equipment or training, and it is a simple clinical assessment tool. The aim of this study was to investigate the relationship between a quantitative ultrasound (QUS) parameter of bone strength and TUGT in Japanese community-dwelling elderly women. [Methods] Ninety-nine community-dwelling elderly women participated (mean age, 74.4 \pm 5.7 years) in the present study. The speed of sound (SOS) in the right calcaneus was measured using a QUS device to assess bone strength. Physical function examinations included grip strength, single leg stance time, TUGT, and gait speed. The Tokyo Metropolitan Institute of Gerontology Index of Competence (TMIG-IC) was used to assess each subject's functioning, and each subject's age, height, and weight were also collected. [Results] In multiple linear regression analysis controlling for age, of the physical function parameters, only TUGT was significantly related to SOS in all subjects. Furthermore, the relationship between SOS and TUGT was significant for those with high TMIG-IC scores, but SOS was not associated with any physical function parameters (including TUGT) for those with low TMIG-IC scores. [Conclusion] TUGT may contribute to the development of a simple clinical assessment tool that could be used to screen for hip fracture risk.
\end{abstract}

Key words: Timed up and go test, Quantitative ultrasound, Community-dwelling elderly women

(This article was submitted Jul. 6, 2009, and was accepted Aug. 6, 2009)

\section{INTRODUCTION}

Decreased bone strength and physical function are known as risk factors of hip fracture in elderly women ${ }^{1)}$. Hagino et al. reported that the incidence of hip fracture has been increasing in elderly women in Japan ${ }^{2}$. The prevention of hip fractures is important for maintaining independence of activities of daily living (ADL) and quality of life for elderly Japanese women, and the appropriate assessment of bone strength and physical function is necessary for the prevention of hip fracture.

To assess bone strength, measurement of bone mass using dual energy X-ray absorptiometry (DEXA) or quantitative ultrasound (QUS) is generally adopted. It has been demonstrated that the assessment of bone strength of the calcaneus using QUS is useful as a predictor of hip fracture ${ }^{3)}$. 
It has been reported that each 1 standard deviation (SD) decrease in the speed of sound (SOS) in the calcaneus, which is one of the QUS indices, increases the risk of hip fracture 2.5 times ${ }^{4)}$.

Of the physical function measures, gait speed has been shown to be an independent predictor of hip fracture $^{3,5)}$. Furthermore, many previous studies have shown that gait speed is related to bone strength assessed by DEXA or QUS ${ }^{6-10)}$. It is also known that falls cause over $90 \%$ of hip fractures ${ }^{11)}$, and the timed up and go test (TUGT) is a useful predictor of falls in the elderly ${ }^{12)}$. However, no previous study has investigated the relationship between bone strength and TUGT. TUGT requires no special equipment or training, and it can be easily measured as part of a clinical examination ${ }^{13)}$. Therefore, determining the relationship between bone strength and TUGT may contribute to demonstrating the usefulness of the TUGT as a predictor of hip fracture. The aim of the present cross-sectional study was to investigate the relationship between QUS parameters of bone strength and TUGT in community-dwelling elderly Japanese women.

\section{SUBJECTS AND METHODS}

\section{Subjects}

The subjects were 99 community-dwelling elderly women. They were recruited by mail or telephone from Gunma prefecture and Kanagawa prefecture, Japan: 49 women from Gunma prefecture, and 50 women from Kanagawa prefecture. The inclusion criteria were: age, 65 years or older; able to walk independently; and no restrictions of physical activities. The exclusion criteria were: known history of neuromuscular disease; myocardial infarction; chronic obstructive pulmonary disease; liver disease; kidney disease; hyperthyroidism; unstable diabetes mellitus; unstable hypertension; taking of prednisolone; or treatment for osteoporosis. Written informed consent was obtained from all the participants. This study was approved by the research ethics committee of J.F. Oberlin University.

\section{Methods}

The SOS in the right calcaneus was measured using a QUS device (CM-100; Furuno Electric Co., Ltd., Hyogo, Japan). When the SOS was measured, the subjects sat on a chair in their bare foot. All measurements were performed using the same device after the same calibration operation, following the operation manual prepared by Furuno Electric Co., Ltd. The device had an accuracy of $\leq \pm 1 \%$ in the $\%$ coefficient of variation.

Physical function examinations consisted of grip strength, single leg stance time, the timed up and go test (TUGT), and gait speed. The grip strength of the right hand was measured using a dynamometer (GRIP-D, T.K.K 5401, Takei Scientific Instruments Co., Ltd., Niigata, Japan) in the standing position. When the single leg stance time was measured, the subjects kept their eyes open, and the time that a subject could stand on her right leg without hand support or falling was measured using a stopwatch. The upper limit of measurement was set at 60 seconds. For TUGT, examiners instructed subjects to stand up from a chair without hand support, walk $3 \mathrm{~m}$ as quickly and safely as possible, turn around, walk back, and then sit down again, as previously reported by Shumway-Cook et al. ${ }^{12)}$. The time required to complete the task was measured using a stopwatch. For gait speed, a $9 \mathrm{~m}$ flat, straight walkway was marked at $2 \mathrm{~m}$ from both ends, for acceleration and deceleration, and gait time was measured between the two points using a stopwatch under two conditions: subjects' preferred speed; subjects' fastest speed possible. The time was divided by the distance each subject walked, and the gait speed was calculated. The Tokyo Metropolitan Institute of Gerontology Index of Competence (TMIG-IC) ${ }^{14)}$ was used to assess the functioning of each subject. This index consists of 13 items, and it has been widely used to measure the extent of elderly persons' functioning. A higher score indicates higher functioning, and the maximum score on this index is 13 points. In addition, age, height, and weight were collected for each subject, and the body mass index (BMI) was calculated based on the height and weight measurements.

The mean value and SD were calculated for each measurement parameter. The relationships between SOS and age, height, weight, BMI, and each physical function parameter were assessed using Pearson correlation coefficients. Furthermore, stepwise multiple linear regression analysis was performed to determine the relationships between SOS and each physical function parameter. In addition, further analysis was performed to investigate the influence on the subject's functioning level. The subjects were divided into two groups by 
Table1. Characteristics, speed of sound and physical function parameters in all subjects

\begin{tabular}{lccc}
\hline & $\begin{array}{c}\text { All subjects } \\
(\mathrm{n}=99)\end{array}$ & $\begin{array}{c}\text { good-functioning } \\
(\mathrm{n}=64)\end{array}$ & $\begin{array}{c}\text { poor-functioning } \\
(\mathrm{n}=35)\end{array}$ \\
\cline { 2 - 4 } & Mean $\pm \mathrm{SD}$ & Mean $\pm \mathrm{SD}$ & Mean $\pm \mathrm{SD}$ \\
\hline Age (years) & $74.4 \pm 5.7$ & $72.7 \pm 4.8$ & $77.5 \pm 6.1^{* *}$ \\
Height $(\mathrm{cm})$ & $148.0 \pm 6.4$ & $149.3 \pm 5.4$ & $145.3 \pm 7.4^{* *}$ \\
Weight $(\mathrm{kg})$ & $51.7 \pm 7.9$ & $51.9 \pm 7.8$ & $51.3 \pm 8.1$ \\
BMI $\left(\mathrm{kg} / \mathrm{m}^{2}\right)$ & $23.6 \pm 3.2$ & $23.3 \pm 3.1$ & $24.3 \pm 3.2$ \\
TMIG-IC $($ full marks $=13$ points) & $12.1 \pm 1.6$ & $\mathrm{NA}$ & $10.3 \pm 1.8$ \\
Speed of sound $(\mathrm{m} / \mathrm{min})$ & $1475.8 \pm 20.5$ & $1478.9 \pm 20.7$ & $1470.1 \pm 19.1^{*}$ \\
Single leg stance time $(\mathrm{sec})$ & $21.7 \pm 19.9$ & $27.2 \pm 20.2$ & $11.6 \pm 15.1^{* *}$ \\
Grip strength $(\mathrm{kg})$ & $22.0 \pm 4.1$ & $22.5 \pm 3.8$ & $21.2 \pm 4.5$ \\
Timed up and go test $(\mathrm{sec})$ & $6.7 \pm 1.9$ & $6.1 \pm 1.3$ & $7.8 \pm 2.4^{* *}$ \\
Gait speed at preferred $(\mathrm{m} / \mathrm{min})$ & $79.3 \pm 12.8$ & $82.5 \pm 11.5$ & $73.4 \pm 13.1^{* *}$ \\
Gait speed at fastest $(\mathrm{m} / \mathrm{min})$ & $100.8 \pm 18.1$ & $107.0 \pm 15.7$ & $89.5 \pm 16.8^{* *}$ \\
\hline
\end{tabular}

good-functioning: TMIG-IC score of full marks (13 points). poor-functioning: TMIG-IC score less than 13 points. TMIG-IC ${ }^{\dagger}$ : Tokyo Metropolitan Institute of Gerontology Index of Competence, NA = Not applicable

$*$ : $\mathrm{p}<0.05$, Comparison with good-functioning elderly, $* *: \mathrm{p}<0.01$, Comparison with good-functioning elderly

the TMIG-IC scores: those who had full-marks (13 points) on the TMIG-IC, "good-functioning", and those who had less than 13 points, "poorfunctioning". Unpaired t-tests were used to compare the age, height, weight, BMI, SOS, and each physical function between the groups. Then, the relationships between the SOS and physical function parameters were analyzed separately for each group using Pearson correlation coefficients and stepwise multiple linear regression analysis. The statistical analyses were performed using SPSS for Windows, version 11.0J (SPSS Japan, Inc., Tokyo, Japan). The significance level was set at $5 \%$.

\section{RESULTS}

The mean values and SDs of the subjects' characteristics, SOS, and physical function parameters are presented in Table 1. There were significant negative correlations between SOS and age $(\mathrm{r}=-0.421, \mathrm{p}<0.01)$ and TUGT $(\mathrm{r}=-0.410$, $\mathrm{p}<0.01)$. There were significant positive correlations between SOS and gait speed at the preferred speed $(\mathrm{r}=0.346, \mathrm{p}<0.01)$, gait speed at the fastest speed $(\mathrm{r}=0.391, \mathrm{p}<0.01)$, and TMIG-IC $(\mathrm{r}=0.198, \mathrm{p}<0.05)$ (Table 2). Stepwise multiple linear regression analysis including age as a covariate was performed, because SOS and physical function were affected by age. In multiple linear regression analysis, of the physical function parameters, only TUGT was significantly related to SOS (Table 3 ). In other words, a slower TUGT was associated with a decreased SOS. However, this result may have been affected by the subjects' functioning level, therefore, further analysis was performed after subjects had been divided into two groups by the TMIG-IC scores. The further analysis demonstrated that elderly subjects with poor-functioning had lower physical function and SOS than elderly subjects with good-functioning (Table 1). With respect to the relationship between SOS and physical function parameters, SOS was significantly correlated with gait speed at the preferred speed $(\mathrm{r}=0.375, \mathrm{p}<0.01)$, gait speed at the fastest speed $(\mathrm{r}=0.426, \mathrm{p}<0.01)$, and TUGT $(\mathrm{r}=-0.595, \mathrm{p}<0.01)$ of good-functioning elderly. Furthermore, the relationship between SOS and TUGT was significant in stepwise multiple linear regression analysis controlling for age. However, SOS was not associated with any of the physical function parameters, including TUGT, in poor-functioning elderly (Table 2 ).

\section{DISCUSSION}

The present study investigated the relationships between a QUS index and TUGT in communitydwelling elderly Japanese women. Previous studies have reported that physical function is associated with a QUS index or BMD using DEXA. Associations of BMD with gait speed, single leg stance time, and muscle strength have been shown in middle-aged and elderly women ${ }^{6,8,15}$ ). 
Table 2. Relationship between speed of sound and characteristics and physical function parameters

\begin{tabular}{lccc}
\hline & $\begin{array}{c}\text { All subjects } \\
(\mathrm{n}=99)\end{array}$ & $\begin{array}{c}\text { good-functioning } \\
(\mathrm{n}=64)\end{array}$ & $\begin{array}{c}\text { poor-functioning } \\
(\mathrm{n}=35)\end{array}$ \\
\cline { 2 - 4 } Independent variable & $\mathrm{r}$ & $\mathrm{r}$ & $\mathrm{r}$ \\
\hline Age & $-0.421^{* *}$ & $-0.500^{* *}$ & -0.192 \\
Height $(\mathrm{cm})$ & 0.105 & 0.074 & 0.029 \\
Weight $(\mathrm{kg})$ & 0.086 & -0.011 & 0.265 \\
BMI $\left(\mathrm{kg} / \mathrm{m}^{2)}\right.$ & 0.044 & -0.044 & 0.321 \\
TMIG-IC $($ points/13) & $0.198^{*}$ & $\mathrm{NA}$ & 0.110 \\
Single leg stance time $(\mathrm{sec})$ & 0.171 & 0.186 & -0.118 \\
Grip strength $(\mathrm{kg})$ & 0.169 & 0.164 & 0.111 \\
Timed up and go test $(\mathrm{sec})$ & $-0.410^{* *}$ & $-0.595^{* *}$ & -0.171 \\
Gait speed at preferred $(\mathrm{m} / \mathrm{min})$ & $0.346^{* *}$ & $0.375^{* *}$ & 0.170 \\
Gait speed at fastest $(\mathrm{m} / \mathrm{min})$ & $0.391^{* *}$ & $0.426^{* *}$ & 0.184 \\
\hline
\end{tabular}

good-functioning: TMIG-IC score of full marks (13 points). poor-functioning: TMIG-IC score less than 13 points. $\mathrm{r}=$ Pearson correlation coefficient, $\mathrm{NA}=$ Not applicable, Dependent variable: speed of sound,

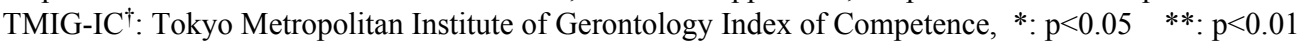

Table 3. Stepwise multiple linear regression analysis (determination of related factors to SOS)

\begin{tabular}{lcccc}
\hline Independent variable & $\mathrm{R}^{2}$ & $\beta$ & $\mathrm{t}$-value & $\mathrm{p}$ value \\
\hline (constant) & & & 60.993 & 0.001 \\
Age (fixed) & 0.177 & -0.276 & -2.485 & 0.015 \\
Timed up and go test (sec) & 0.042 & -0.250 & -2.254 & 0.026 \\
(excluded independent variable) & & & -1.001 & 0.320 \\
Single leg stance time (sec) & & -0.106 & 0.950 & 0.345 \\
Grip strength (kg) & 0.090 & 0.227 & 0.821 \\
Gait speed at preferred (m/min) & & 0.031 & 0.333 & 0.740 \\
Gait speed at fastest (m/min) & & 0.054 & -0.765 & 0.446 \\
TMIG-IC
\end{tabular}

Dependent variable: speed of sound, $\mathrm{TMIG}^{-\mathrm{IC}^{\dagger}}$ : Tokyo Metropolitan Institute of Gerontology Index of Competence.

Furthermore, in studies involving communitydwelling elderly women, muscle strength and gait speed were related to BMD or QUS ${ }^{7,10)}$. With respect to walking ability, Kwon et al. reported that a decline in gait speed was associated with decreased BMD in a longitudinal observation of community-dwelling elderly women ${ }^{9}$. Furthermore, Kitagawa et al. reported a relationship between the number of daily walking steps and QUS ${ }^{16,17)}$. The results of these previous studies demonstrate that walking ability is related to BMD and QUS, but the relationship between BMD or QUS and TUGT was not investigated in these previous studies. As in previous studies, the present study also showed that gait speed was related to QUS in univariate analysis. However, only TUGT was significantly related to QUS in multivariate analysis.

TUGT is associated with gait speed and $\mathrm{ADL}^{13)}$. Furthermore, TUGT is known to be useful for predicting falls in the elderly and in patients after hip fracture surgery ${ }^{12,18)}$. Also, Morris et al. reported that TUGT was more useful than gait speed as a predictor of falls in community-dwelling elderly women with a history of vertebral fracture ${ }^{19)}$. Therefore, TUGT can be considered a comprehensive assessment tool of physical function and fall risk for the elderly. The relationship between TUGT and QUS in multivariate analysis found in the present study may be responsible for feature of TUGT that reflect physical function and fall risk of elderly women. Besides, the relationship between QUS and the TUGT found in the present study is of great interest from the standpoint of fall 
causation in hip fractures.

A straight walkway of at least $10 \mathrm{~m}$ is required to measure gait speed. In addition, a pedometer and a certain period of time are required to examine the number of daily walking steps. Therefore, measurements of gait speed and daily walking steps are less practical than TUGT at the local community level, because TUGT requires a distance of only $3 \mathrm{~m}$ and no special equipment or training. Thus, TUGT is more clinically practical than gait speed or daily walking steps. The present findings concerning the relationship between QUS and TUGT may prove helpful in the development of a simple clinical assessment tool for screening for the risk of hip fracture.

In the present study, no relationship between SOS and TUGT was found for the poor-functioning elderly. The reason for this result cannot be clearly determined based on the data of the present study alone. It has been suggested that several factors are responsible for bone strength. For example, bone strength is associated with body weight, physical activity, and calcium intake in elderly women ${ }^{20)}$. Indeed, the present study found a weak correlation between SOS and BMI in poor-functioning elderly, though the correlation was not statistically significant (Table 2). Physique, as measured by BMI or body weight, may have more influence on bone strength than physical function in poorfunctioning elderly.

The present study had several limitations. Since the present study was a cross-sectional design, the cause and effect relationship between TUGT and QUS cannot be determined. The association between fracture and the TUGT was not investigated; therefore, the usefulness of the TUGT as a predictor of fracture should be carefully interpreted. Finally, the factors (physical function, calcium intake, and BMI) associated with SOS cannot be clearly demonstrated in poor-functioning elderly, since the sample size of poor functioning elderly was small in the present study. Therefore, further data on SOS and physical function in the elderly with poor function need to be accumulated.

\section{REFERENCES}

1) NIH Consensus Development Panel on Osteoporosis Prevention, Diagnosis, and Therapy: Osteoporosis prevention, diagnosis, and therapy. JAMA, 2001, 285: 785-795.
2) Hagino $H$, Katagiri $H$, Okano $T$, et al.: Increasing incidence of hip fracture in Tottori prefecture, Japan: Trend from 1986 to 2001. Osteoporos Int, 2005, 16 : 1963-1968.

3) Dargent-Molina P, Schott AM, Hans D, et al.: Separate and combined value of bone mass and gait speed measurements in screening for hip fracture risk: results from the EPIDOS study. Osteoporos Int, 1999, 9: 188192.

4) Fujiwara S, Sone T, Yamazaki K, et al.: Heel bone ultrasound predicts non-spine fracture in Japanese men and women. Osteoporosis Int, 2005, 16: 2107-2112.

5) Dargent-Molina P, Favier F, Grandjean H, et al.: Fallrelated factors and risk of hip fracture: the EPIDOS prospective study. The Lancet, 1996, 348: 145-149.

6) Aoyagi K, Ross PD, Hayashi T, et al.: Calcaneus bone mineral density is lower among men and women with lower physical performance. Calcif Tissue Int, 2000, 67: 106-110.

7) Taaffe DR, Simonsick EM, Visser M,et al.: Lower extremity physical performance and hip bone mineral density in elderly black and white men and women: cross-sectional associations in the health ABC study. $\mathrm{J}$ Gerontol Med Sci, 2003, 58A: 934-942.

8) Lindsey C, Brownbill RA, Bohannon RA, et al.: Association of physical performance measures with bone mineral density in postmenopausal women. Arch Phys Med Rehabil, 2005, 86: 1102-1107.

9) Kwon J, Suzuki T, Yoshida H, et al.: Association between change in bone mineral density and decline in usual walking speed in elderly community-dwelling Japanese women during 2 years of follow-up. J Am Geriatr Soc, 2007, 55: 240-244.

10) Sun $W$, Watanabe $M$, Tanimoto $Y$, et al.: Ultrasound parameters of bone health and related physical measurement indicators for the community-dwelling elderly in Japan. Geriatr Gerontol Int, 2007, 7: 154159.

11) Norton R, Campbell AJ, Lee-Joe T, et al.: Circumstances of falls resulting in hip fractures among older people. J Am Geriatr Soc, 1997, 45: 1108-1112.

12) Shumway-Cook A, Brauer S, Woollacott $M$ : Predicting the probability for falls in communitydwelling older adults using the Timed Up \& Go test. Phys Ther, 2000, 80: 896-903.

13) Podsiadlo D, Richardson S: The timed "up \& go": a test of basic functional mobility for frail elderly persons. J Am Geriatr Soc, 1991, 39: 142-148.

14) Koyano W, Shibata $H$, Nakazato $K$, et al.: Measurement of competence: reliability and validity of the TMIG Index of Competence. Arch Gerontol Geriatr, 1991, 13: 103-116.

15) Bayramoğlu $M$, Sözay $S$, Karataş $M$, et al.: Relationship between muscle strength and bone mineral density of three body regions in sedentary postmenopausal women. Rheumatol Int, 2005, 25: 513-517.

16) Kitagawa J, Omasu F, Nakahara Y: Effect on daily 
walking steps on ultrasound parameters of the calcaneus in elderly women. Osteoporos Int, 2003, 14: 219-224.

17) Kitagawa J, Nakahara Y: Associations of daily walking steps with calcaneus ultrasound parameters and a bone resorption maker in elderly women. $\mathrm{J}$ Physiol Anthropol, 2008, 27: 295-300.

18) Kristensen M, Foss NB, Kehlet H: Timed "Up and Go" test as a predictor of falls within 6 months after hip fracture surgery. Phys Ther, 2007, 87: 24-30.

19) Morris R, Harwood RH, Baker R, et al.: A comparison of different balance tests in the prediction of falls in older women with vertebral fractures: a cohort study. Age Ageing, 2007, 36: 78-83.

20) Uusi-Rasi K, Sievänen $H$, Pasanen $M$, et al.: Maintenance of body weight, physical activity and calcium intake helps preserve bone mass in elderly women. Osteoporos Int, 2001, 12: 373-379. 